\title{
Eye disease in a patient with rheumatoid arthritis
}

\author{
M Shinzato, J Yamamoto, C E Hirata, A C Goldberg, N H Yoshinari, E Bonfá
}

\begin{abstract}
Summary
We report the case of a 40-year-old women with diffuse uveitis, sensorineural hearing loss and cerebrospinal fluid pleocytosis as features of Vogt-Koyanagi-Harada syndrome who developed symmetric polyarthritis and stiffness of small and large joints, in addition to rheumatoid arthritis. Although their target tissues are distinct, both diseases have a possible autoimmune origin strongly associated with HLADRB4.
\end{abstract}

Keywords: Vogt-Koyanagi-Harada syndrome; rheumatoid arthritis; autoimmune disease

Vogt-Koyanagi-Harada (VKH) syndrome is a multi-organ disorder affecting pigmented structures, such as the eye, inner ear and meninges. It can also affect skin. It has been described as an inflammatory condition of autoimmune nature in which cytotoxic $\mathrm{T}$ cells target the melanocytes. ${ }^{1}$ The strong association of $\mathrm{VKH}$ disease with DR4/DR53 in the Japanese population has been known since 1976 and has also been described in patients of Hispanic origin living in Southern California. In these patients, a secondary association to HLA-DR1 was also described, involving the share sequence linked to rheumatoid arthritis. ${ }^{2}$

Rheumatoid arthritis (RA) is a common chronic inflammatory disease characterised by a symmetric sterile and progressive synovitis within joints. Approximately $75 \%$ of patients with RA exhibit a positive rheumatoid factor. After several months or years from disease onset, characteristic bone erosions become apparent on X-rays. It is also associated with HLA-DR4 and DR1 alleles that share a sequence motif in the third hypervariable region, therefore named rheumatoid epitope. ${ }^{3}$ The most common ocular symptom in RA is keratoconjunctivitis sicca, occurring in $9-31 \%$ of patients. The frequency of scleritis is said to be $0.15-6.3 \% .{ }^{4}$ Episcleritis, scleromalacia perforans, and corneal ulceration may also be found. Uveitis is rare in adult RA.

In this paper we report a case of RA associated with VKH syndrome. To the best of our knowledge, such an association has only been described once before.

\section{Case report}

Nineteen years ago, a white 40-year-old woman was admitted with a history of blurred vision, ocular discomfort, photophobia accompanied by headache, dizziness and hearing loss.
She also complained of arthralgias with morning stiffness. On initial examination, her visual acuity was hand movements at both eyes. She had $3+$ cells and $2+$ flare in both anterior chambers. There was $1+$ cells in both anterior vitreous bodies. A bilateral serous retinal detachment was present. Audiometric evaluation revealed sensorineural impairment of both ears. Cerebrospinal fluid (CSF) displayed 112 cells/dl, predominantly of lymphomononuclear origin (96\%); Wassermann and VDRL reactions were negative. Serology for toxoplasmosis was also negative. Haemoglobin level was 13.8 $\mathrm{g} / \mathrm{dl}$, and erythrocyte sedimentation rate (ESR) was $27 \mathrm{~mm}$ in the first hour. The patient was treated with prednisone $1 \mathrm{mg} / \mathrm{kg}$, and showed improvement of ocular disease as well as of the neurologic and otologic symptoms. Although the prednisone reduction was extremely gradual over a period of 10 years, the patient had several recurrences of the ocular manifestations with posterior development of bilateral cataract. Cataract surgery was performed in 1988. The eye diseases remained stable with low dose corticosteroid. Examination of the fundus shows the 'sunset glow' appearance (figure 1) typical of $\mathrm{VKH}$ syndrome.

Over the next 4 years, she developed polyarthritis of large and small joints with morning stiffness lasting for an hour. She presented poor motion and symmetric arthritis of wrists, metacarpophalangeal, proximal and distal interphalangeal joints, and also of the right knee and ankle. Laboratory tests disclosed hypochromic, normocytic anaemia (haemoglobin $10 \mathrm{~g} / \mathrm{dl}$ ). The ESR was $56 \mathrm{~mm}$ in the first hour. Her polyclonal gammaglobulin level was high

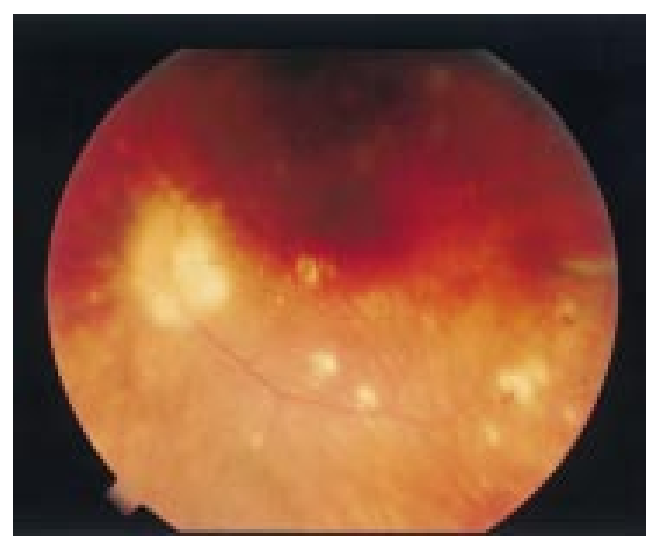

Figure 1 The 'sunset glow' appearance of the fundus and retinal pigment epithelium from resolved Dalen fuchs nodules in a convalescent phase of $\mathrm{VKH}$ syndrome 


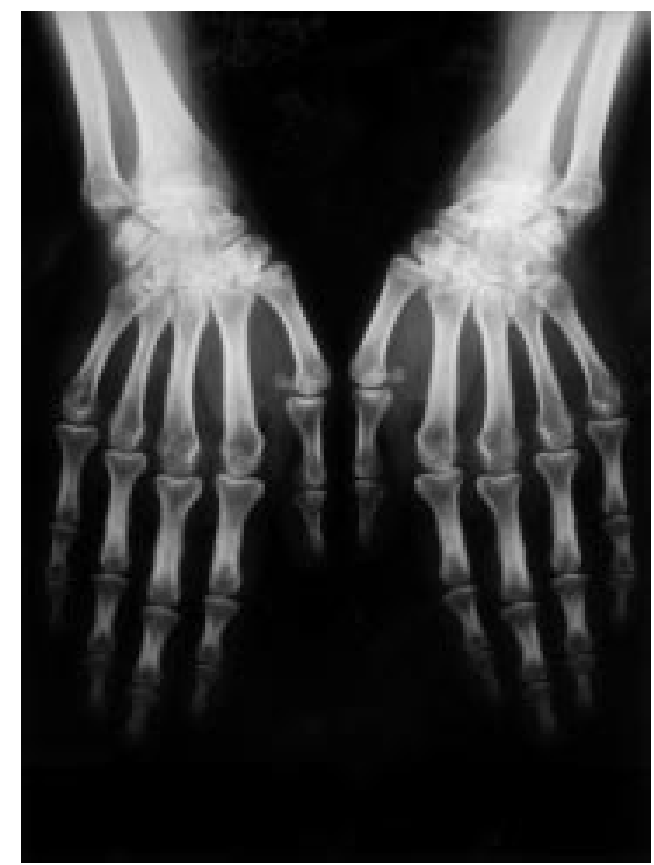

Figure 2 X-Ray of the patient's hands showing a symmetric peri-articular osteopenia, cartilage narrowing and bone erosions, especially in the wrists

on protein electrophoresis. Rheumatoid factor (RF) was positive. Her HLA class II profile was DR4 (DRB1*0401), DR11，DR52，DR53, DQ7, DQ8. Radiography of the patient's hands (figure 2) shows peri-articular osteopenia, cartilage narrowing and bone erosions, especially in the wrists.

\section{Discussion}

The exudative retinal detachment panuveitis without a history of eye trauma associated with pleocytosis of CSF and sensorineural impairment of ears confirmed the diagnosis of

1 Moorty, RS, Inomata, H, Rao, NA. Vogt-Koyanagi-Harada syndrome. Surv Ophthalmol 1995; 39: 265-92.

2 Weiz, JM, Holland, GN, Roer LN, et al. Association between Vogt-Koyanagi-Harada syndrome and HLA-DR1 and -DR4 in Hispanic patients living in southern California. Ophthalmology 1995;102:11012-5.

3 Gregerson, PK, Silver, J, Winchester, RJ. The share epitope hypothesis: an approach to understanding the molecular genetics of susceptibility to rheumatoid arthritis. Arthritis Rheum 1987;30:1205-13.

4 Matsuo T, Kono R, Matsuo N, et al. Incidence of ocular complications in rheumatoid arthritis and the relation of keratoconjuntivitis sicca with its systemic activity. Scand $f$ Rheumatol 1997;26:113-6.

5 Egashira K, Yamamoto T, Tsuji S, Onishi A, Muray Y. A case of myastenia gravis complicated with chronic rheumatoid arthritis and Vogt-Koyanagi-Harada disease. Rinsho Shinkeigaku 1987;27:859-61.

\section{Learning points}

- rheumatoid arthritis and Vogt-Koyanagi-Harada syndrome can occur concomitantly

- they are both supposed to have an autoimmune nature

- although they are both associated with HLA-DR4, they have different target tissues

Vogt-Koyanagi-Harada syndrome according to the criteria established by the American Uveitis Society. ${ }^{6}$ Dermatological findings described in $\mathrm{VKH}$ are alopecia, poliosis and vitiligo, but our patient did not have any of these cutaneous manifestations. On the other hand, she presented with arthralgia and morning stiffness which have not been described in this disease. In fact, later, this patient developed a symmetric erosive polyarthritis, including an elevated ESR and positivity to rheumatoid factor fulfilling the American Rheumatism Association criteria for RA. ${ }^{7}$ The finding of HLA-DRB $1{ }^{\star} 0401$ further supports this diagnosis.

The aetiology of VKH is unknown but its autoimmune nature is suggested by the occasional association with disease such as polyglandular syndrome type $\mathrm{I}^{8}$ and scleroderma. ${ }^{9}$ The association presented herein is particularly interesting since the two conditions are associated with HLA-DR4 alleles which share the sequence linked to RA. Although the most common allele in $\mathrm{VKH}$ is $\mathrm{DRB} 1^{\star} 0405$, pocket 4 is very similar in $\mathrm{DRB} 1{ }^{\star} 0401$ and 0405 and outlines the peptide-binding motif for RA. ${ }^{10}$

In conclusion, this report describes a rare case of RA-associated VKH syndrome. Although the real occurrence of VKH among the RA population is unknown because of the rarity of the former, this report presents an opportunity to establish an eventual relationship between these two diseases of autoimmune origin.

6 Snyder DA, Tessler HH. Vogt-Koyanagi-Harada syndrome. Am f Ophthalmol 1980;90:69-71.

7 Arnett FC, Edworthy SM, Block DA. The American Rheumatism Association 1987 revised criteria for the classification of rheumatoid arthritis. Arthritis Rheum 1988;31: $558-9$.

8 Jovic NS, Nesovic M, Vransevic DN, Ciric J, Marincovic DM, Bonaci B. The Vogt-Koyanagi-Harada syndrome association with autoimmune polyglandular syndrome type association with autoimmune poly
1. Postgrad Med f 1996;72:495-7.

9 Cavalli C, Gobbi PG, Colombo R, Ricardi A, Gorini M, Ascari E. Un cas de sclérodermique et association au syndrome de Vogt-Koyanagi-Harada. Presse Méd 1985;14: $1131-4$.

10 Hammer J, Gallazi F, Bono E, et al. Peptide-binding specificity of HLA-DR4 molecules: correlation with rheumatoid arthritis association. $\mathcal{F}$ Exp Med 1995;181:1847-55. 